\title{
EL LENGUAJE DE LAS ROTULACIONES DE ESTABLECIMIENTOS COMERCIALES EN LAS CIUDADES CONTEMPORÁNEAS. LOS CASOS DE ALMERÍA, LÓDŹ Y TARRASA
}

\author{
THE LANGUAGE OF THE MARKINGS OF COMMERCIAL \\ ESTABLISHMENTS IN THE CONTEMPORARY CITIES. \\ THE CASES OF ALMERÍA, ŁÓDŹ AND TARRASA
}

\section{Francisco GARCÍA MARCOS}

Universidad de Almería fgarcos@gmail.com

\begin{abstract}
Resumen: El artículo analiza el paisaje lingüístico de la cartelería pública en las ciudades de Almería, Łódź y Tarrasa (Barcelona). En concreto, se ocupa de los letreros comerciales relacionada con el deporte. Se ha elegido el deporte por ser uno de los grandes referentes sociales en la actualidad. Ese análisis contrastivo demuestra el predominio de nombres internacionales. Esa tendencia supera incluso la legislación lingüística que defiende el uso de lenguas vernáculas. Se trata, pues, de una situación que reproduce los procesos de globalización del mundo actual. La experiencia empírica ha permitido también tratar algunas cuestiones teóricas y metodológicas relacionadas con el estudio del paisaje lingüístico.
\end{abstract}

Palabras clave: Paisaje lingüístico. Semiosis urbana. Sociolingüística. 


\begin{abstract}
The article analyses the linguistic landscape of public signage in the cities of Almería, Łódź and Tarrasa (Barcelona). In particular, it deals with commercial signs related to sport The sport has been chosen because it is one of the great social referents nowadays. Contrastive analysis shows the predominance of international names. This tendency even exceeds the linguistic legislation that defends the use of vernacular languages. It is, then, a situation that reproduces the globalization processes of the current world. The empirical experience has also allowed to deal with some theoretical and methodological issues related to the study of the linguistic landscape.
\end{abstract}

Key Words: Linguistic landscape. Urban semiosis. Sociolinguistics.

\title{
1. INRODUCCIÓN. SEMIOSIS URBANA
}

La concepción de cualquier ámbito humano como un gran espacio de múltiples significaciones no es nueva. En sustancia ese ha sido un planteamiento siempre latente en la moderna semiótica, constituyendo uno de los grandes puntos de común acuerdo entre sus principales formulaciones nucleares (Barthes, 1971; Eco, 1964, 1968; Greimas, 1976; Lotman, 1973 1979, 1984, Serrano, 1979, 1993). Cualquier entorno está repleto de signos $\mathrm{y}$, en consecuencia, transmite múltiples significados. Lo hace de forma explícita unas veces, pero en otras ocasiones esos contenidos significativos viajan implícitos a través de un sinfín de detalles, bien de los signos en su conjunto, bien entre alguno de sus componentes. Todos estos signos llegan a los ciudadanos que los descodifican, los interpretan y actúan conforme a la información que reciben de ellos.

Ese entramado, en cualquier caso, no se dispone de manera azarosa. Muy al contrario responde a las principales pautas de comportamiento e identidad colectivos de una determinada sociedad (Eco, 1964). Aproximarse 
a sus signos supone adentrarse en los valores y actividades que los nutren, de los que surgen y a los que retroalimentan. Ese es el propósito genérico de esta investigación, concentrada en uno de los signos más frecuentes de la iconografía urbana, los carteles identificativos de sus establecimientos y edificios.

El lenguaje tiene su aportación relevante en esa semiótica urbana, incluso con un área propia asociada a las competencias más recientes de la sociolingüística. Los estudios de linguistic landscape (de paisaje lingüístico, $L L$ o $P L$ en su versión española) se ocupan de cómo las lenguas se hacen visibles dentro de los entornos físicos de las sociedades. Su campo de estudio abarca, sin restricciones, cualquier manifestación verbal escrita para el público urbano: señales, pancartas, vallas, pósteres, letreros en diferentes soportes (luminosos, fijos, etc.), instrucciones de uso del mobiliario público, publicidad, afiches, reclamos en los escaparates, grafiti, carteles y rótulos de instituciones o de entidades privadas.

Tal amplitud casuística solo podía generar un número no menos extenso de posibles agentes condicionantes de la configuración última que adopte un determinado paisaje lingüístico. Ese nuevolistado ha contemplado la configuración diglósica de sociedades con lenguas en contacto, la implantación de estructuras coloniales, las migraciones, el despliegue de procesos de internacionalización, su contrapartida localista deseosa de conservar sus raíces vernáculas o las acciones políticas encaminadas a recuperar el espacio social de las lenguas minoritarias. Esa abundante heterogeneidad precisaba ser acotada para resultar mínimamente operativa a la hora de acometer la investigación empírica. Lo más inmediato fue caracterizar las unidades de análisis como signos. Establecido ese marco general, Franco Rodríguez (2005) lo especificó en el texto: Sin apartarse de su conceptualización como signo, sí precisó de qué tipo de signo en concreto se trataba. Al mismo tiempo, convenía determinar en qué clases podrían agruparse esas unidades básicas, al objeto de trazar una mínima tipología del PL Ben Rafael (2009) propuso una primera diada que fue aceptada de inmediato en la bibliografía, discriminando entre lo que 
llamó "Top-down" (los emitidos por entidades públicas) y "Bottom-up" (los de personas concretas o compañías). Franco Rodríguez (2013), por su parte, matizó de nuevo esa propuesta, adoptando una perspectiva más dinámica, muy útil para el trabajo empírico, que cruzaba actores (privados, corporativos e institucionales) con el radio de acción de las unidades (local, regional, nacional e internacional).

De ese modo se conseguía ensamblar un corpus teórico y unas mínimas bases metodológicas, que desarrollasen las primeras formulaciones del $P L$, ya presentes en la sociolingüística norteamericana con la que concluye la centuria pasada (Landry y Bourhis 1997:23; Spolsky y Cooper 1991). A partir de ahí se ha ido incorporando una cada vez mayor masa empírica, con investigaciones en ciudades como Los Ángeles (Franco, 2005, 2011), Tokyo (Backhaus 2006, 2007), Bangkok (Huebner 2006), Miami (Franco, 2005), Jerusalén (Ben-Rafael et al. 2006), Madrid (Muñoz, 2010; Castillo y Sáez, 2011), Sevilla (Pons, 2012 o, entre otras, Almería (Franco, 2013). Tampoco han faltado panorámicas generales como la de Shohamy y Gorter (2009).. No obstante, a pesar de esos avances sustanciales, en fechas relativamente recientes Sebba (2010:73) lamentaba todavía una cierta indefinición, o cuando menos que no se hubieran resuelto de manera satisfactoria todos los problemas planteados. En cualquier caso,, ello no ha impedido que el $P L$ estableciera un perfil científico propio, no exento de coincidencias con otras investigaciones más delimitadas, dentro y fuera de la sociolingüística. A través de Written Europe varias escuelas de diseño del Viejo Continente (Ámsterdam, Riga, Praga, Madrid 10) estudian la tipografía urbana, siguiendo el interés del Buchstabenmuseum de Berlín, encargado de preservar y conservar letreros con valor histórico o cultural. Parten del postulado de que la rotulación urbana trascribe la dinámica de toda sociedad, lo que supone en la actualidad confrontar la dialéctica identidad vernácula vs. estandarización globalizadora (González Riaza, 2006; Gamonal, 2011 para el caso de Madrid).

La sociolingüística de la cartelería urbana comparte algunos de esas inquietudes e incorpora otras. Interesa relativamente el tipo 
caligráfico y el soporte, pero sobre todo el contenido verbal de los letreros, su ubicación urbana y las lenguas empleadas en los mismos, además de las funciones semióticas que pueden desempeñar, básicamente informativas, connotativas, simbólicas y persuasivas.

Todo ello aconsejaría, sin duda, una profusa investigación, que excedería los limites razonables de este trabajo. Aquí se persiguen objetivos bastante más modestos, operando con una triple focalización epistemológica, temática y espacial, al margen de centrarse únicamente en la rotulación de los establecimientos (no en el conjunto de $P L$ ). De un lado, se desarrollará dentro de los límites del lenguaje, abordando tanto la selección de lenguas empleadas, como los contenidos de los nombres de establecimientos públicos. De otro, se concentrará en un solo ámbito temático, el deporte, bien es cierto que contrastado en todo momento con el resto de epígrafes comerciales que cubre la cartelería pública. Y, por último, restringirá sus observaciones a tres comunidades, en principio muy diferenciadas entre sí, las ciudades españolas de Almería y Tarrasa (Barcelona), junto a la polaca Łódź.

El deporte es uno de los grandes iconos sociales, y por tanto semióticos, de la sociedad contemporánea. Espectáculo de masas por excelencia en nuestros días, asequible para una inmensa mayoría de ciudadanos (García Ferrando, 1986: 108) o, entre otras cosas, amalgamador de las relaciones intergrupales (Greendorfer 1981), alcanza una relevancia psicosocial de tal calibre que más del $60 \%$ de los padres encuestados en España por García Ferrando aspiraban a que sus hijos e hijas fuesen deportistas profesionales. Súmese a ello que es una de las consecuencias más directamente vinculadas al desarrollo de las llamadas sociedades postmodernas (Águila Soto, 2005). Lo hace, además, incorporando una nueva vertiente considerablemente distinta de la anterior, como hábito o patrón de vida, unas veces inmerso en la actividad física en sentido amplio, otras en niveles deportivos alejados de la gran profesionalización.

De una u otra forma, o en ambas simultáneamente, todo ello se transcribe en importante cuota de presencia en los medios de comunicación, 
incremento del consumo de material deportivo, abundancia de publicidad y de tópica social en torno a la actividad física, el deporte y a sus ídolos. También lo hace en excelentes balances económicos, que empezaron a repuntar desde finales de los 80 (Moati, 1990), siendo capaces de sortear con eficiencia la última gran crisis económica mundial. Manejando estadísticas deportivas del bienio 2014-2015, elaboradas por del Ministerio de Educación, Cultura y Deportes español, Blázquez (2015) recuerda que el sector deportivo ha generado 24.000 M€.

Abordar tres comunidades como Almería, Tarrasa (Barcelona) y la ciudad polaca de Łódź, en última instancia enlaza con una de las hipótesis apuntadas tanto desde las investigaciones sobre paisaje lingüístico, como desde el proyecto Written Europe. La rotulación y la cartelería comerciales pueden ser exponentes privilegiados de tendencias globalizadoras y locales, de estandarización uniformadora y de idiosincrásica vernácula, máxime en una temática tan extendida y universal como el deporte.

Esas tres comunidades -Almería, Łódź, Tarrasa- están suficientemente diferenciadas, aunque sin acusar excesivos hiatos entre ellas, sobre todo en lo tocante a su historia inmediata y a la complejidad de sus redes sociales. Almería, con 194203 habitantes (Censo de 2015), ubicada en el SE de la Península Ibérica dentro de la Comunidad Autónoma Andaluza, ha experimentado una profundísima transformación socioeconómica durante las tres últimas décadas. De ser una de las zonas más deprimidas del Estado Español, ha pasado a convertirse en el epicentro de la agricultura industrial del Mediterráneo; también en una comunidad multiétnica y multicultural con una fuerte presencia de inmigración. Ello ha tenido la lógica repercusión en todos los órdenes, incluido el idiomático. Cuenta, además, con una excelente incursión en su paisaje lingüístico (Franco Rodríguez, 2013), bien es verdad que con más diversificados intereses temáticos y más concentrada en tres áreas urbanas.

Tarrasa, en la comarca barcelonesa del Vallés Occidental, cuenta con 215214 según el mismo Censo de 2015. En las últimas tres décadas ha reorientado igualmente su tejido productivo, evolucionando desde la 
tradicional industria textil hacia nuevas formas de negocio, tanto en el sector secundario como en el terciario. Con una presencia constante de población inmigrada durante la última centuria, desde el punto de vista idiomático ha formado parte de una comunidad autónoma con bilingüismo oficial (catalán y español), junto con numerosas lenguas importadas de sus últimos procesos inmigratorios. Como todas las poblaciones de Cataluña, y en relación directa al objetivo central de este trabajo, está sujeta a normativas explícitas sobre consumo y política lingüística, las leyes 22/2010, de 20 de julio y 1/ 1998, de 7 de enero. En relación a la rotulación y a la cartelería, establecen que siempre deberán estar escritas como mínimo en catalán, en lo que se interpreta como una clara acción de normalización lingüística de esta lengua dentro de su ámbito autonómico.

Łódź, con 718.960 habitantes oficiales en 2012, por su parte, es una gran urbe tradicional polaca, ubicada en el centro geográfico del país, lo que la convierte en un enclave logístico de primera magnitud. Esa, precisamente, ha sido una de sus principales transformaciones económicas durante las últimas tres décadas, sustituyendo de ese modo su arraigado sector textil. Es lugar completamente monolingüe, siguiendo por lo demás una pauta común a todo el dominio polaco, donde la diversidad dialectal está muy atenuada. En realidad, se limita al casubio (en Pomerania, al Norte del país), al mazoviano (en Mazuria, en la región de los lagos, también al Norte) y al silesio (región de Podhale, en los Tatras limítrofes con Eslovaquía) ${ }^{1}$.

\section{METODOLOGÍA}

Fijados esos tres puntos de encuesta, se han realizado calas sistemáticas desde 2014, aplicando técnicas de inmersión etnográfica, fruto de las cuales se recogió de manera indiscriminada toda clase de cartelería

\footnotetext{
${ }^{1}$ Quiero dejar constancia de mi gratitud hacia el Prof. Dr. M. Baran (Universidad de Łódź) por aclararme, con su habitual magisterio, la cuestión dialectal polaca, no tan evidente en la bibliografía.
} 
de establecimientos. El trabajo de campo permitió confeccionar muestras muy homogéneas, con 1737 entradas en Almería, 1869 en Tarrasa y 1980 en Łódź. De esa manera se ha conformado un corpus amplio y ostensiblemente diversificado, tanto como en definitiva es la propia vida urbana: desde rotulaciones de nombres de autoescuelas, consultas odontológicas, bares y cafeterías, hasta la delegación de un ministerio o, en fin y entre un listado mucho más amplio, un taller mecánico.

No ha sido siempre fácil, ni inmediato, encontrar parámetros que permitiesen ordenar tanta diversidad ni, sobre todo, que delimitasen los factores que condicionarían la aparición de un tipo u otro de cartel. Es cierto que, como se ha visto antes, las investigaciones en $P L$ habían avanzado algo en esa dirección, pero en todo caso no terminaba de ajustarse a todo el material del supuesto concreto que aquí se examina. La diferencia entre lo público y lo privado prácticamente se neutraliza, dada la abrumadora mayoría de la segunda clase de cartelería. Otro tanto sucede con el parámetro de localización, utilísimo para otros ámbitos del $P L$, aunque mucho más limitado en el caso que aquí se trata. A fin de cuenta, casi todos los establecimientos están encuadrados dentro de la categoría local. Existen, y no serán desatendidos, cadenas nacionales e internacionales que, sin embargo, estadísticamente no dejan de ser testimoniales, si bien adquieren una alta relevancia cualitativa. Para resolver esas dificultades se propusieron seis parámetros más directamente inmediatos a la idiosincrasia de la realidad examinada, con independencia de que alguno de ellos pudiera resultar subespecificado a posteriori para ajustar su precisión analítica. De esa manera cada entrada fue etiquetada con su carácter público o privado, el alcance del establecimiento (nacional, local, etc.), su nombre, la lengua (o lenguas) empleadas en su rotulación, su ubicación dentro de la ciudad y el producto -o los servicios- que ofrecía a los ciudadanos. Los dos primeros epígrafes proceden de la bibliografía del $P L$. Los dos siguientes no supusieron mayores inconvenientes. Tanto el nombre $-\mathrm{O}$ nombres- de los establecimientos reflejados en las rotulaciones, como las lenguas empleadas en ellos, evidentemente, son referencias objetivas que 
se obtienen de manera directa durante el trabajo de campo. No sucede otro tanto ni con la ubicación urbana de esos establecimientos ni, en especial, con los productos o servicios que ofrecen.

Ha sido relativamente delicado establecer una tipología de este último punto, de entrada, por su propia heterogeneidad, pero también por la ausencia de criterios uniformes entre la bibliografía especializada. Desde los estudios empresariales se discrimina el radio de venta de los establecimientos (mayorista/minorista), su filiación comercial (negocio propio/cadena/franquicia) o su grado de especialización (gran superficie/ detall). Todas ellas, sin duda, son magnitudes pertinentes para aproximarse a la fisonomía de la empresa comercial. Pero, en todo caso, no son útiles para abordar con garantías la relevancia semiótica de los recursos empleados por los establecimientos en su ubicación dentro del paisaje urbano a través de sus carteles. Desde el marketing, por su parte, al menos sí se percibe cierta proximidad a los objetivos de esta investigación. En primer lugar, porque se opera a partir de una primera división que discrimina entre productos dedicados al consumo, productos dedicados a los negocios y productos clasificados en función de su duración y tangibilidad. De esa primera división genérica deriva otra ostensiblemente más específica, conformada por los 45 epígrafes que contempla la Clasificación Internacional de Productos y Servicios de Niza (2012). Gracias a ello se cuenta con un criterio, bastante objetivo, pero sobre todo homologado por la práctica administrativa y jurídica de la UE. Ese ha sido el referente fundamental desde el que se han elaborado aquí las subespecificaciones contempladas en lo relativo a la tipología de servicios y productos ofrecidos a través de la cartelería urbana de los establecimientos, al que se le han agregado las correcciones cualitativas necesarias, sobre todo, para hacerla más operativa.

Con todo, ha sido una tarea no exenta de dificultades de cierta envergadura. Para empezar, la Clasificación de Niza contempla, como por otra parte era de esperar, toda clase de actividades, entre las que se incluyen las empresas de producción industrial básica (fábricas químicas, 
de industria pesada, etc.). Por descontado que estas exceden el marco de este trabajo, concentrado en los comercios del ámbito urbano, y no tanto en cualquier rotulación empresarial de forma indiscriminada. A continuación, cada uno de esos epígrafes, a su vez, se desgrana en un número considerable de subespecificaciones empresariales (el 1 tiene 13 subapartados, el 2 otros 10, etc.) Además, hay una considerable transversalidad en los listados de Niza, dado que pueden encontrarse distintos productos (desgranados en los 45 epígrafes) que se comercialicen sin embargo en un solo tipo de establecimiento ${ }^{2}$. Por último, no se contempla nada referido a la administración que englobe toda la rotulación oficial, sin duda un componente fundamental de la semiótica urbana.

Ante ese denso panorama, se corría el más que plausible riesgo de emplear una taxonomía demasiado prolija, y en consecuencia poco útil, dispersa por lo demás en lo cuantitativo y en lo cualitativo. Ante esa evidente dificultad, se optó por reformular la Clasificación de Niza, al objeto de que sea científicamente operativa en estudios de este tipo, atendiendo a los siguientes criterios:

1. Se prestará únicamente atención a los nombres de comercios y servicios que conforman la cartelería urbana. Quedan excluidos, por tanto, las entidades dedicadas a la producción en otros sectores y en otras ubicaciones.

2. Se incluye la comunicación pública de la administración

3. Se procederá a agrupar temáticamente productos y servicios comercializados de forma homogénea, con independencia de que puedan proceder de uno o varios apartados de la Clasificación de Niza.

4. La anterior agrupación, acorde con el conjunto del panorama semiótico de la ciudad, no excluye que un estudio monográfico de cada uno de ellos requiera de sucesivas subespecificaciones que, en todo caso, exceden el marco de este trabajo.

\footnotetext{
${ }^{2}$ Por ejemplo, en una papelería habría productos comercializados desde la clase 01 (pegamento), 16 (clips), 28 (papel de manualidades), etc.
} 
Aplicando esos criterios, se determinaron finalmente 23 áreas temáticas, todas ellas vinculadas a varios apartados de la Clasificación de Niza, excepto en el caso de la administración, incluida por las razones que acabo de señalar ${ }^{3}$. El deporte ocupa el epígrafe 05, aunando productos y servicios contemplados en los epígrafes 09, 25, 28, 41 de la Clasificación de Niza. Para proceder a su análisis se han contemplado tres subespecificaciones básicas, discriminando entre establecimientos comerciales (tiendas deportivas), centros de actividad física y deportiva (gimnasios, escuelas de buceo, etc.) y clubes y entidades deportivas. Todas ellas entrarían dentro de la categoría de bottom-up privadas, admitiendo, en principio, los cuatro radios de alcance propuestos por Franco Rodríguez, esto es, ser de carácter local, regional, nacional o internacional. A esos tres grandes grupos habría que agregar un cuarto, no por su peso cuantitativo, pero sí por la relevancia sociolingüística de los nombres de entidades y edificios públicos destinados al deporte, presentes en el paisaje lingüístico de las tres ciudades. El Patronato Municipal de Deportes de Almería, el Circuit Municipal de Ciclisme de Terrassa o la Miejski Szkolny Związek Sportowy, la escuela deportiva municipal de Łódź, forman parte de una rotulación pública de alto contenido sociolingüístico. En total, unos y otros, el mundo del deporte muestreado ha arrojado 57 entradas en Almería, 70 en Łódź y 65 en Tarrasa, lo que supone el 3’28\%, 4’09\% y 3’47\% de sus respectivas muestras.

En cuanto a la distribución espacial de ese muestreo, se han considerado cuatro clases de zonas urbanas, que por lo demás se corresponden con una división clásica y cualitativamente sociológica de las ciudades contemporáneas, dejando al margen los posibles anejos que

${ }^{3}$ El listado final queda conformado por los siguientes centros temáticos: 01 administración, 02-alimentación, 03-arte y cultura, 04-automoción, 05-deporte, 06-educación, 07-estética, 08-gestión y finanzas, 09-consumo personal, 10-hogar, 11-hostelería, 12-informática, 13-juguetería, 14-mantenimiento, 15-multiproductos, 16-ocio y recreo, 17-papelería, 18-religión y espiritualidad, 19-ropa y complementos, 20-sanidad, 21-sexo, 22-telefonía y 23 -transportes. 
puedan tener incorporados sus términos municipales. En sentido general, se sigue la filosofía de las investigaciones sociológicas sobre diferenciación residencial (Sweetser, 1965; Timms, 1971), aunque excluyendo su análisis factorial que excedería con mucho esta investigación. De esa manera, los materiales de la muestra han quedado encuadrados entre las siguientes subespecificaciones espaciales:

01- Núcleo urbano, donde radica el grueso de la ciudad, definido en términos negativos respecto de los otros tres apartados; esto es, no es ni centro histórico, ni periferia ni extrarradio.

02- Centro histórico, constituido por el casco histórico y zonas inmediatamente adyacentes, donde además se concentra la mayor parte de la administración oficial y una gran actividad comercial y financiera.

03- Periferias urbanas, zonas de expansión de las ciudades, con frecuencia destinadas a usos residenciales, con reducción de servicios, aunque estos mantengan un alto nivel.

04- Extrarradio urbano, compuesto por barrios marginales que han crecido alrededor de los núcleos consolidados, generalmente habitados por los sectores más deprimidos de la población.

Los dos primeros asumen la división espacial empleada por Franco (2013) en su análisis en Almería, mientras que se incluyen los dos siguientes pretendiendo abarcar el conjunto del ámbito urbano.

Por lo demás, es previsible que todos los factores contemplados actúen de forma integrada, de manera que el contenido de una rotulación y la selección de la lengua en que se verbaliza sean fruto de la posible interacción combinada, por una parte, de su ubicación en el mapa urbano y, por otra, del tipo de producto y/o servicio ofrecidos. En todo caso, ello no deja de ser una hipótesis que en última instancia le compete abordar -y, en su caso, tratar de aclarar- a este trabajo. 


\section{ANÁLISIS}

Para empezar, la presencia de rotulación deportiva tiene una relevancia análoga en las tres comunidades. Al establecer un listado de áreas temáticas ordenado por rangos ${ }^{4}$ de frecuencias de aparición, el deporte se mueve entre posiciones bastante equivalentes, tal y como refleja el cuadro siguiente.

\begin{tabular}{|c|c|c|c|c|c|c|c|}
\hline \multirow[t]{2}{*}{ Área } & \multicolumn{3}{|c|}{ Rangos } & \multirow[t]{2}{*}{ Área } & \multicolumn{3}{|c|}{ Rangos } \\
\hline & Alm & $\mathbf{L z}$ & Te & & Alm & Lz. & Ter \\
\hline 01 administración & 13 & 12 & 10 & 13-juguetería & 15 & 9 & 12 \\
\hline 02-alimentación & 1 & 1 & 1 & 14-mantenimiento & 20 & 5 & 19 \\
\hline 03-arte y cultura & 16 & 13 & 14 & 15-multiproductos & 21 & 17 & 21 \\
\hline 04-automoción & 8 & 18 & 6 & 16-ocio y recreo & 17 & 11 & 13 \\
\hline 05-deporte & 12 & 14 & 11 & 17-papelería & 22 & 10 & 20 \\
\hline 06-educación & 7 & 6 & 5 & 18-religión/espíritu & 4 & 2 & 4 \\
\hline 07-estética & 9 & 15 & 8 & 19-ropa/compl. & 3 & 4 & 3 \\
\hline 08-gestión/finanzas & 5 & 7 & 7 & 20-sanidad & 23 & 23 & 23 \\
\hline 09-personal & 18 & 16 & 17 & 21-sexo & 6 & 21 & 9 \\
\hline 10-hogar & 10 & 8 & 15 & 22-telefonía & 11 & 22 & 22 \\
\hline 11-hostelería & 2 & 3 & 2 & 23-transportes & 19 & 19 & 18 \\
\hline 12-informática & 14 & 20 & 16 & & & & \\
\hline
\end{tabular}

Alm: Almería; Lz.: Łódź; Te: Tarrasa.

Tabla 1. Rangos de áreas temáticas en los nombres de establecimientos en Almería, Łódź y Tarrasa.

El deporte oscila entre el rango 11 de Tarrasa y el 14 de Łódź, con el 12 de Almería, siempre por tanto en posición intermedia en la lista de 23 rangos de cada ciudad. Esa posición, en todo caso, tampoco está exenta de algunas matizaciones muy significativas. Todas las áreas temáticas contempladas aquí, y subsidiariamente en la Clasificación de

${ }^{4}$ Esto es, del más frecuente (rango 1) al que menos ha aparecido en la muestra (rango 23). 
Niza, tampoco comparten la misma relevancia sociológica desde el punto de vista cualitativo. De un lado, hay establecimientos que remiten a necesidades perentorias e inexcusables. Que la alimentación, la educación, la ropa o la sanidad ocupen invariablemente los primeros rangos se desprende de lo vital de sus productos y servicios para la vida cotidiana. De otro, hay establecimientos regulados legalmente-caso de las farmacias o de los estancos en España-, cuya distribución queda al margen de otras valoraciones; cumplen con una ratio de distancia y número de habitantes $\mathrm{y}$, en consecuencia, su distribución espacial está regulada por ley. Finalmente, quedan los negocios, con su correspondiente rotulación y cartelería, que sí recogen hábitos de consumo, estilos de vida, preferencias sociales o, en sentido amplio, tendencias colectivas; también, al mismo tiempo, reflejan en gran medida la idiosincrasia de una comunidad ${ }^{5}$. Al margen de todo condicionamiento coyuntural, se encuentran los rótulos oficiales, los religiosos y los de grandes superficies. Los primeros dependen de la propia estructura de la Administración, los segundos siguen las necesidades de culto de los fieles y no están sujetos a mercadotecnia y, en cuanto a los últimos, cuentan con una presencia mucho más puntual, como corresponde a un establecimiento excepcional que amalgama otros en su interior.

\subsection{Cartelería deportiva en Almería, Lódź y Tarrasa. Perspectiva general}

Los relacionados con la actividad física y el deporte se encuadran dentro del tercer epígrafe, el que he denominado como "negocios". En el seno de ese grupo restringido sí muestran posiciones relativas considerablemente altas. Lo hacen, además, de una manera permanente y bastante homogénea en los tres puntos analizados, lo que pondría de manifiesto que se trata de una constante transversal de la sociedad contemporánea, común a los núcleos urbanos incluidos dentro de la dinámica globalizadora. Hay, en

${ }^{5}$ Por ejemplo, establecimientos dedicados a la venta de gorras en el Norte de Alemania, de instrumental de esquí en Suiza o de playeras en el Mediterráneo. 
todo caso, particularidades muy acusadas, que parecen estar vinculadas a la idiosincrasia de cada contexto, sobre todo en función de la tipología interna de la actividad deportiva. En la muestra han aparecido tres grandes clases de rotulaciones relacionadas con ella, tal y como se había previsto de partida, al margen de los establecimientos oficiales: las referentes a establecimientos de material deportivo, las de centros de actividad física y las de clubes que, para empezar, muestran diferentes cuotas de presencia, además de los organismos oficiales.

Tipo

Ciudades

\begin{tabular}{ccccccc} 
& Almería & \multicolumn{2}{c}{ Lódź } & \multicolumn{3}{c}{ Tarrasa } \\
\cline { 1 - 5 } & $\mathrm{N}$ & $\%$ & $\mathrm{~N}$ & $\%$ & $\mathrm{n}$ & $\%$ \\
\hline Clubes & 14 & $24^{\prime} 5$ & 32 & $45^{\prime} 7$ & 16 & $24^{\prime} 6$ \\
Tiendas & 19 & $33^{\prime} 3$ & 14 & $20^{\prime} 0$ & 22 & $33^{\prime} 8$ \\
Centros & 24 & $42^{\prime} 1$ & 24 & $34^{\prime} 2$ & 26 & $39^{\prime} 9$ \\
$\mathrm{~N}$ & 57 & & 70 & & 65 &
\end{tabular}

Tabla 2. Porcentajes de rotulaciones deportivas según tipo de establecimiento en las comunidades de Almería, Łódź y Tarrasa.

En el cuadro anterior se apuntan -como mínimo de forma tendencialdos patrones nacionales, probablemente vinculados a hábitos de consumo en primera instancia, pero también a organización de la actividad física y deportiva. En Almería y Tarrasa las cifras prácticamente coinciden en términos porcentuales. Hay un ligero incremento de rotulación procedente de centros de actividad física en Almería, explicable por su historia reciente. Esa ciudad fue sede de los Juegos Mediterráneos en 2005, lo que entre otras cosas significó una ostensible presencia de infraestructura deportiva que ha permanecido dentro del paisaje urbano. Tarrasa, por su parte, cuenta con instalaciones deportivas de mayor recorrido cronológico, asentados en la ciudad y con el consiguiente circuito comercial asociado a ello. En todo caso, las diferencias más notables, como he apuntado, hace 
un instante, aparecen al contrastar estos datos con Łódź. En la ciudad polaca predominan los clubes deportivos y, por el contrario, descienden de manera notable las tiendas especializadas en materia deportiva.

\subsection{Clubes}

La terminología de los carteles, por otra parte, incorpora información muy estimable acerca del rol del deporte en las sociedades europeas contemporáneas, en primera instancia, pero también por la propia imagen semiótica que construye de sí mismo, sobre todo a través del lenguaje que selecciona. Todo ello queda recogido en la tabla 3.

En Almería los nombres más clásicos -quizá también los más convencionales-recurren a referencias concretas sobre la práctica deportiva de la que se ocupa el club y la localidad a la que representa (Club Ciclista Ciudad de Almería), pudiéndose agregar un patrocinador consolidado por años de vinculación (Club Voleibol Unicaja de Almería). Naturalmente, existen acrónimos como el de URA (Unión Rugby Almería) y referencias históricas muy vernáculas (Club Balonmano $\left.U r c i^{6}\right)$. En todo caso, dentro de la capital y sus núcleos poblacionales inmediatos, predomina de forma absoluta el español, a diferencia de lo que sucede en las vecinas comarcas costeras, donde si es posible encontrar denominaciones inglesas, casos de Happy Kayak, Sun Golf Gotia o Desert Springs Golf Club. Esta opción está claramente vinculada al reclamo turístico, buscando un referente internacional de comunicación. De ahí que el caso almeriense muestre dos prototipos directamente asociados al rol semiótico de esta nomenclatura. Por un lado, el español permite desempeñar una función básicamente referencial; esto es, apelar directamente a la actividad (el deporte) y su identificación verbal (el nombre del club). Por otro, la terminología inglesa es más propicia para fines comerciales directamente asociados al turismo, que combinan recreo con prácticas deportivas susceptibles de ser incluidos

${ }^{6}$ Urci debió ser un asentamiento íbero, todavía no localizado, sobre cuya localización se debate hoy en día. 
en esos paquetes (casos del golf o del kayak), con una presencia inmediata y significativa en la publicidad de esos productos.

En Tarrasa, la normalización sociolingüística en catalán se ha cumplido, aunque solo en parte, por motivos que trascienden a la mera rotulación de los establecimientos deportivos. Parte de la nomenclatura de los propios clubes sigue fuera de ese proceso. De las 181 entidades, asociaciones y clubes deportivos que oficialmente recoge el ayuntamiento de la ciudad ${ }^{7}, 111$ usan completa o parcialmente el catalán. Resta más de un 30\% que mantiene su nombre en español, al margen de otra cuota significativa de entidades y clubes recogidos en lenguas extranjeras. En ambos supuestos la casuística es muy variada. El actual Club Handbol Terrassa se fundó en 1953 como San Fernando de Tarrasa, un nombre cargado de simbología franquista como patrón de la juventud. En la Transición, a principios de los 80, se eliminó esa referencia tan connotada, sustituyéndola por un cambio de nombre y un cambio de lengua (Handbol). No fue un caso único, por supuesto. El histórico y laureado Club d'Escacs Terrassa también tuvo su ancestro en español, capaz de organizar grandes torneos, muy reconocidos por la prensa de la época, como los de 1945 o 1960. Otro tanto sucedió, por no extenderme en la casuística, con el Club Natació Terrassa, otro de los emblemas deportivos de la ciudad, con versión en español durante el Franquismo. Por supuesto, tras la normalización a partir de los años 80 se ha acudido a nombres catalanes para otros clubes de creación más reciente, caso del Club de Rugby Carboners de Terrasssa, fundado en 2009. Bien es verdad que en algunas ocasiones la grafía de ambas lenguas coincide (Club Egara, hockey), lo que ha hecho innecesarias acomodaciones de este tipo. Junto a ellas subsiste esa cuota significativa de clubes en español (Club Deportivo Pueblo Nuevo). No existe un patrón regular que condicione la elección de la lengua en función del tipo de actividad deportiva. De hecho, se registran ambas en muchas asociaciones dedicadas a un mismo campo (Sant Pere Nord Club de Fútbol y Peña San Pedro Club de Fútbol, ambos en la misma barriada).

${ }^{7}$ Cf. http://www.terrassa.cat/es/llista-d-entitats-04 [20/12/2017] 
Al margen de la selección de lengua, como en el caso de Almería se incluyen nombres con referencias directas a la práctica deportiva que realizan (Terrassa, F. C.), con acrónimos (JABAC, fútbol; EPIC-Casino del Comerç), con extranjerismos, fundamentalmente de origen inglés (Champions Trophy Terrassa 2006) u oriental (Club Esportiu Goshindo $U M A$ ), algunos de ellos con ortografía catalana normalizada (Associació Esportiva de Tai Txi Yuyan) y, por último, de nuevo con referencias históricas a la antigua Egara, villa romana fundada en época de Vespasiano, entre el 69 y 79 a.C., (además del equipo de hoceky, Club Agility Egara, Club Balonkorf Egara'85, Cluba Handbol Egara, Peña ciclista Egara, Sociedad de Pescadores deportivos Egara). Los acrónimos han mostrado especial ductilidad y acomodación a los cambios de política lingüística. El $J A B A C$ se fundó en 1950, con el nombre de Juventud Atlética Balompédica Amateur. Una década después se agregó “Carmelitano", debido a su fusión con el Colegio Nuestra Señora del Carmen, en cuyos terrenos estaba construido el campo de fútbol que utilizaban. En 2006 llevan a cabo una segunda fusión, esta vez con el Terrassa F. C., con un nuevo terreno de juego en el extremo sur de la ciudad y, también, con un nuevo escenario lingüístico, al que se acomoda sin dificultad: se persiste el acrónimo, sin detallar en qué consisten las siglas, aunque manteniéndolas para no perder la referencia histórica del club, envueltas en terminología catalana, dando por resultado Unió de Futbol Base Jàbac ${ }^{8}$ i Terrassa. Otras veces las reconversiones fueron más inmediatas. Para el antiguo EPYC (Entidad Polideportiva y Cultural) bastó con cambiar la ortografía de la conjunción copulativa ("y" española por la "i" catalana) para completar la operación de normalización lingüística en EPIC.

En Łódź, en cambio, predomina mayoritariamente el polaco en los nombres de sus clubes más señeros, empezando en el fútbol con el Widzew Łódź y continuando con el Lódzkiego Sportowego Towarzystwa Waterpolowego de waterpolo o con el ŁSTW AZS UŁ PŁ Anilana Łódź de balonmano, fruto de múltiples gestiones y de no poco tesón para que se

${ }^{8}$ Curiosamente se aplican las reglas de acentuación del catalán a un acrónimo en español. 
mantuviera activo. La notación polaca alcanza prácticamente las tres cuartas partes de los clubes, dejando menos del 30\% para la aparición de términos, como en los dos casos anteriores, de procedencia sajona (Berserker's Team Łódź, Sport Perfect FootballAcademy, Wake Up Fitness) u oriental (Weng Chun Kung Fu Łódź, Ving Tsun Kuen Kung Fu). Hay, por lo tanto, una mayor homogeneidad en la terminología polaca, frente a la dispersión que se registra en España, bien sea a consecuencia del bilingüismo de algunas zonas (Tarrasa), bien al incremento del uso de lenguas extranjeras.

\begin{tabular}{|c|c|c|c|}
\hline Lengua & Almería & Łódź & Tarrasa \\
\hline Español & $83^{\prime} 63$ & - & $23^{\prime} 16$ \\
\hline Catalán & - & - & $42^{\prime} 71$ \\
\hline Polaco & - & $82^{\prime} 53$ & - \\
\hline Inglés & 4'15 & $3{ }^{\prime} 42$ & $5^{\prime} 15$ \\
\hline Lenguas. orientales & 2'14 & 3’05 & $1^{\prime} 62$ \\
\hline Español/inglés & $6 ’ 21$ & - & 4'38 \\
\hline Catalán/inglés & - & - & $8^{\prime} 94$ \\
\hline Polaco/inglés & - & 7’19 & - \\
\hline Español/1. orientales & 3'85 & - & $5^{\prime} 66$ \\
\hline Catalán/1. orientales & - & - & 8'19 \\
\hline Polaco/1. orientales & - & 3'81 & - \\
\hline $\mathrm{N}$ & 15 & 32 & 37 \\
\hline
\end{tabular}

Tabla 3. Distribución porcentual de lenguas en la denominación de entidades deportivas de Almería, Łódź y Tarrasa. 
Más allá del predominio de los idiomas vernáculos, las lenguas orientales se imponen en rotulaciones mixtas en las tres comunidades. Temáticamente se circunscriben única y exclusivamente a los deportes procedentes de esa parte del planeta que, por lo general, carecen de referentes para ser traducidos en lengua de recepción. El inglés, en cambio, se distribuye conforme a un gradatum que va de Tarrasa a Łódź, pasando por Almería, pero que en todo caso atestigua una evidente internacionalización. Es cierto que en algunos casos está muy vinculado a prácticas deportivas importadas, novedosas y con posible repercusión positiva para el turismo (Almería). Pero, al margen de situaciones específicas, ejerce también como gran referente internacional. De hecho, en las comunidades administrativamente monolingües, Łódź y Almería, los porcentajes son muy similares, como también sucede con las lenguas orientales, lo que parece indicar con claridad que se trata de tendencias que van más allá del ámbito estatal, o en su caso de la coyuntura particular de cada comunidad de habla.

A la vista de ello, es evidente que de entre todos los posibles agentes que condicionan el $P L$, solo son activos los que tienen que ver con la fuerte -y constante- contraposición entre vernacularidad e internacionalización. Los nombres de los clubes deportivos parecen inmunes a la localización concreta donde se encuentran sus respectivas sedes sociales en las tres localidades examinadas. De un lado, se impone su voluntad de identificación por encima del factor ubicación. De otro, esta, la ubicación, no deja de ser algo coyuntural que, en consecuencia, no debe afectar a la identidad del club. Las sedes pueden trasladar sus domicilios, estos a su vez pueden variar de estatus dentro de la ciudad, factores todos ellos que en última instancia no afectan a la identidad del club.

Tampoco se manifiesta ninguna influencia de radio de alcance. Son clubes locales, radicados en cada una de sus respectivas ciudades, sin dependencia directa de otros ámbitos regionales, nacionales o locales. 


\subsection{Tiendas de deportes}

Los centros comerciales, por su parte, muestran un comportamiento lingüístico considerablemente diferente. En el Cuadro-4, para empezar, desaparece por completo la terminología oriental como reclamo de venta de material deportivo, así como los letreros mixtos. Se prefieren mensajes mucho más directos, inequívocamente identificativos que, en consecuencia, eviten cualquier forma de ruido que pueda desorientar al consumidor. Para continuar, abren, aunque sea testimonialmente, el catálogo de lenguas empleadas, en la mayoría de las ocasiones para subrayar los vínculos semióticos con su especialidad deportiva. Así, en Almería existe Eolo un establecimiento deportivo, un referente en material de windsurfing, aunque no sea su única especialidad. En Tarrasa existe Charpoua, para montañeros como corresponde al mítico refugio ubicado en la vertiente francesa del Mont-Blanc. En Łódź hay una referencia italiana, Sportisimo, menos marcada que las dos anteriores. Por último, en las tres comunidades se reiteran grandes firmas internacionales, casos de Decathlon o Intersport. En esta ocasión sí actúa con claridad el alcance de los establecimientos, dado el carácter internacional de estas cadenas de tiendas deportivas. No lo hace la migración, otro de los posibles condicionamientos del $P L$ ni en Almería ni en Tarrasa. En ambas ciudades, sí aparecen nombres en algunas de las últimas lenguas llegadas a ellas, especialmente en árabe, pero en lo que antes he considerado establecimientos de primera necesidad; esto es, alimentación, ropa o peluquería.

\footnotetext{
${ }^{9}$ Se refiere al dios del viento griego, hijo de Hípotes, que fundó su dinastía en las islas Eolias, al que Zeus le dio poder para controlar los vientos.
} 


\begin{tabular}{l|lll}
\multicolumn{1}{c|}{ Lengua } & Almería & \multicolumn{1}{c}{ Lódź } & \multicolumn{1}{c}{ Tarrasa } \\
\hline Español & $56^{\prime} 2$ & - & $31^{\prime} 2$ \\
Catalán & - & - & $18^{\prime} 7$ \\
Polaco & - & $52^{\prime} 3$ & - \\
Inglés & $37^{\prime} 5$ & $28^{\prime} 5$ & $43^{\prime} 7$ \\
Italiano & - & $4{ }^{\prime} 7$ & - \\
Griego & $66^{\prime 2}$ & - & - \\
Francés & - & - & $66^{\prime 2}$ \\
Polaco/inglés & - & $14^{\prime 2}$ & - \\
$\mathrm{N}$ & 16 & 21 & 16
\end{tabular}

Tabla 4. Distribución porcentual de lenguas empleadas en los establecimientos deportivos de Almería, Łódź y Tarrasa.

Almería mantiene una polarización muy acusada entre inglés y español. Hay una pequeña incidencia de rotulaciones mixtas, caso de Ochoruedas Online Skate Shop Almería. El inglés parece fuertemente asociado a especializaciones deportivas relativamente modernas (Footbubble Bodywork Mare Sport). La parte española, en cambio, es de más amplio espectro, abarca desde denominaciones muy ceñidas a una determinada práctica deportiva (Depor Pesca), hasta establecimientos con una larga tradición en la ciudad y un propietario reconocido (Deportes Blanes), incluyendo connotaciones claras sobre actividad física y deportiva (Corre Corre, Décimas) y localizaciones topográficas vernáculas (Deportes Andarax). La ubicación de los establecimientos comerciales en esta ocasión tampoco ha sido determinante, distribuyéndose estos parámetros a lo largo de toda la geografía de la ciudad.

Algo bastante análogo sucede en Łódź, donde el uso del polaco es preferente con bastante holgura (Sklep sportowy e-sportowy, Korona), siguiendo a continuación el inglés (Avalon Sportwear). A más distancia se encuentran rótulos mixtos polaco/inglés (GO Sport Łódź Galeria Łódzka) 
en mayor o menor grado. Aparte de la notación italiana, se observa cierta tendencia a las siglas $(U p 8,4 f)$. La ubicación sí parece ser pertinente en Łódź sobre todo para los establecimientos en inglés, y especialmente para los más vinculados a las grandes cadenas internacionales, sobre todo al quedar ubicados en Manufaktura, el gran centro neurálgico de actividad comercial en la ciudad. Es más, en no poca medida Manufaktura ha sido un símbolo de comercio y modernidad dentro de Łódź, por lo que cobra especial relevancia esa terminología sajona.

Entre las de Tarrasa, en cambio, predominan con mucha distancia los establecimientos con nombres en inglés, más allá de la política de normalización lingüística de la Generalitat. Son denominaciones muy específicas y directamente conectadas con la actividad físico-deportiva en la que están especializadas, como I Love Padel, Kulture Shop. A continuación, siguen los que incluyen rotulaciones en español (Deportes Moya, Tiendasmil), incluso por delante de los catalanes (Cercle Sport). En esta ocasión, la ubicación si es condicionamiento significativo. El español y el catalán están más asentados en el núcleo urbano y en las barriadas más periféricas. El inglés, dentro de su predominio general, se intensifica sobremanera en el centro histórico. Esa es una tendencia, por lo demás, común a toda clase de establecimientos tarrasenses. Lo mismo sucede con el comercio de ropa, la gastronomía, los complementos y, en general, cualquier actividad comercial.

\subsection{Centros de actividad física}

Los centros de actividad física conforman el apartado más complejo de los contemplados aquí por su propia heterogeneidad interna, no siempre equiparable entre sí, como indica el Cuadro-5. De un lado, incluye lugares más tradicionales, los gimnasios, que en la actualidad acogen un número muy diversificado de actividades. De otro, hay establecimientos más especializados en deportes, o en familias de deportes, mucho más restringidos, como serían las artes marciales o los centros de fitness. Por 
último, hay entidades, en un sentido más moderno del término, capacitadas para abarcar muchas actividades, desde las antes mencionadas, hasta piscinas o pistas polideportivas. Como se verá de inmediato, ello tiene repercusiones muy directas y vinculantes sobre la elección del nombre elegido para mostrarse a la sociedad.

\begin{tabular}{l|cll} 
Latín & $3^{\prime} 7$ & - & - \\
L. orientales & $18^{\prime} 5$ & $20{ }^{\prime} 8$ & $11^{\prime} 5$ \\
Español/inglés & $11^{\prime} 1$ & - & - \\
Español/Orientales & $7{ }^{\prime} 4$ & - & $3{ }^{\prime} 8$ \\
Catalán/inglés & - & - & $3{ }^{\prime} 8$ \\
Polaco/inglés & - & - & $3{ }^{\prime} 8$ \\
Nombres propios & $3{ }^{\prime} 7$ & $4{ }^{\prime} 1$ & $11^{\prime} 5$ \\
\hline $\mathrm{N}$ & 27 & 24 & 26
\end{tabular}

Tabla 5. Distribución porcentual de lenguas empleadas

en los centros de actividad física y deportiva de Almería, Łódź y Tarrasa

Como rasgo compartido por los tres núcleos de población analizados, sobresale el uso de nombres propios como estrategia comercial, identificando el conjunto del establecimiento con el prestigio de las personas que se encargan de ellos. Ese recurso ha sido relativamente abundante en Tarrasa, donde es posible encontrar centros de estas características como los de Victoria Gali Marsiñac, Quirós Granados o Angels Llonch Viñas. Tampoco son desconocidos en Łódź, caso del dirigido por Tańca Jankowski.

Los centros en español predominan con cierta holgura en el conjunto del listado de Almería. En ocasiones se apela a referencias un tanto genéricas (Centro 45), a denominaciones generales de una entidad con más actividades (Club Costa) o a rótulos que buscan obviamente implicar al ciudadano (Almería y tú). El fitness se muestra más propicio al 
inglés (Station Fitness, eBody), aunque otras prácticas y actividades físicas también acuden a esta lengua (Olympic, K-sport). Las artes marciales, como cabía suponer, se inclinan más por la terminología oriental, bien en exclusiva (Bushidokan), bien a través de titulaciones híbridas (Shinseiken Dojo Artes Marciales). En cambio, los rótulos que combinan español e inglés tampoco parecen seguir un patrón muy reconocible, habida cuenta de que aparecen incluso en deportes muy arraigados en Almería (Almería Boxing Center) o en establecimientos mucho más generalistas (Nivel Uno Gym). Curiosamente, o significativamente quizá, las instalaciones multiactividad más modernas y avanzadas han recurrido a un término en lengua clásica, el Ego latino, quizá como forma de subrayar su singularidad. Esa diversificación temática es el principal condicionamiento en la selección lingüística de estos rótulos en Almería, por encima de su localización en uno u otro punto de la ciudad.

Łódź mantiene el mismo patrón general, aunque enfatizando la presencia de la lengua vernácula, el polaco de entidades como Duża sala gimnastyczna. El fittness atrae al inglés y su combinatoria con el polaco (Energy Fitness Club, Club Sportera Fitness - Lódź), mientras que las artes marciales, siguiendo la tendencia general, incorporan rasgos de lenguas orientales (Ving Tsun Kung Fu, Bartitsu Kokoro Judo). No obstante, Łódź muestra algunas singularidades bastante acusadas, al menos en determinados aspectos. Conserva cierta inclinación al uso de siglas (ZSS6 Zespól Spól Specjalnych), como ya sucediera en los centros comerciales. Además, dispone de instituciones educativas que habilitan -y eventualmente alquilan- sus instalaciones deportivas fuera del horario escolar (Liceum Mundurowe), Salezjańska Szkoła). Esa dinámica se desenvuelve al margen de lo espacial en el caso de Łódź, entre otros motivos porque tampoco tiene tanto margen de variación interna, dado el uniforme predominio del polaco.

Tarrasa sigue una pauta considerablemente similar a la de Almería, solo que sustituyendo el catalán por el español que, justo en este apartado, presenta sus índices más bajos de aparición en toda la muestra. Predomina 
el catalán (Gimnàs Cos Olimpic), aunque seguido muy de cerca por el inglés (McFit, Perfecctfit), de nuevo ostensiblemente vinculado al fitness. También como en el caso almeriense, las lenguas orientales están vinculadas al aprendizaje, entrenamiento y desempeño en artes marciales (Ken Ze), siendo además la principal fuente de convergencia de lenguas, tanto con el español (Centro de Formación Yuyán), como con el catalán (Associació Cultural Musubi Aikido Terrassa). El español, queda dicho, cuenta con una presencia testimonial, por debajo del inglés e incluso de las lenguas orientales. La ubicación urbana en esta ocasión vuelve a condicionar la selección lingüística. El catalán, y los testimoniales ejemplos de español, se localizan en el centro y el núcleo urbano, mientras que las lenguas foráneas, ambas, se desplazan hacia las barriadas y las periferias.

\subsection{Edificios y entidades deportivas oficiales}

Los nombres de los edificios y entidades deportivas oficiales no escapan a la tónica que, con mayor o menor énfasis, ha recorrido los tres apartados anteriores. En Almería se subraya siempre el carácter institucional de las instalaciones o de las entidades, por lo general apelando a su propiedad municipal (Patronato Municipal de Deportes). Sobre esa base, se suelen agregar referencias topográficas sobre su ubicación (Complejo Deportivo Municipal Distrito 6 Los Ángeles) o, con bastante frecuencia, como homenaje a figuras o acontecimientos señalados de la vida deportiva de la ciudad. Así, existe un Estadio Municipal de los Juegos Mediterráneos, conmemorando la celebración de los mismos en 2005, otro dedicado a Emilio Campra y diferentes Pabellones en memoria de Antonio Rivera o Moisés Ruiz, entre otros.

Las autoridades de Łódź se han mostrado más proclives a una terminología más directa y funcional para referir sus instalaciones deportivas. Sus salas municipales de deportes se rotulan en términos literales como Hala Sportowa Miejskiego Ośrodka Sportu i Rekreacji w $Ł o d z i$, con apenas concesión para indicar su novedad en otras ocasiones, 
Nowa Hala Sportowa Łódź. En esa ciudad la percepción del deporte y el recreo, en sentido más amplio, están unidas, hasta el punto de plasmarse también en la rotulación de sus instalaciones municipales (Miejski Ośrodek Sportu i Rekreacji w Łodzi). Se mantiene también cierta propensión a las siglas, como en otras ocasiones dentro de su PL deportivo, caso de Hala Sportowa MOSiR Łódź MQiSR, Miejski Ośrodek Sportu i Rekreacji). Igual espíritu prosaico se mantiene en el nombre del estadio municipal que cobija al equipo de fútbol de la ciudad (Stadion Widzewa Łódź). Esa tónica solo se rompe con el Atlas Arena, un moderno y multifuncional pabellón polideportivo finalizado en 2009 , de titularidad igualmente municipal.

Los edificios deportivos de propiedad pública sí que aplican con rigor la normativa lingüística catalana en Tarrasa, empezando por el organismo municipal encargado de coordinar la atención al deporte, el Consell Esportiu, y terminando por su Estadi Olimpic Municipal, sede de las competiciones de hockey durante Barcelona'92. En Tarrasa predominan sobre todo las referencias a la localización más inmediata de sus infraestructuras deportivas. De esa manera, a la identificación de las instalaciones ("poliesortiu", "zona esportiva"), sigue de inmediato su pertinente topónimo (Sant Llorenç, Les Arenes, La Maurina, Can Jofresa), correspondiente a las barriadas donde está situado. En comparación con otros ámbitos deportivos, en este sí resulta determinante el carácter oficial del emisor de la rotulación para la selección exclusiva del catalán.

\section{CONCLUSIONES. LENGUAS Y PAISAJE URBANO EN EL ÁMBITO DE LOS DEPORTES}

Los tiempos actuales se desenvuelven entre las coordenadas de un proceso obvio de internacionalización en todos los niveles que, desde luego, encuentra también su transcripción, directa y fehaciente, en el ámbito de la actividad física y del deporte. Se consume deporte a escala global (retransmisiones de ligas fuera de sus países de origen, olimpiadas, grandes eventos), se comercia merchandaising en el mundo entero e incluso 
los fans de los grandes clubes desconocen las fronteras. No es de extrañar, pues, que eso se refleje en sus rotulaciones urbanas más cotidianas. Entre otras cosas, reproducen los mismos iconos y claves semióticas que circulan a través de múltiples canales sociales, reforzando los mensajes anteriores. De ese modo, en su paisaje lingüístico aparecen grandes firmas y cadenas internacionales de comercialización, se manifiesta una acusada inclinación a recurrir al inglés como indicador de modernidad y calidad, y se reserva un espacio concedido a la singularidad de las lenguas orientales. En la rotulación comercial tampoco sucede nada sustancialmente distinto a lo que cualquier ciudadano encuentra en los medios de comunicación, en Internet o en las meras conversaciones entre amigos.

Hay, de todas formas, matices que inciden sobre esa tendencia global que, en apariencia, se aleja de esa línea de tensión entre lo vernáculo y lo foráneo. La complejidad de los contextos sociolingüísticos de base, en primer lugar, puede atenuar ese patrón. En las comunidades multilingües el inglés suele competir con más de una lengua, además de con la legislación lingüística, como sucede en Tarrasa. Sin embargo, su extensión parece inevitable, evidenciando una restricción muy difícil de salvar para las políticas lingüísticas proteccionistas.

El ámbito deportivo, en segundo lugar, también condiciona de manera significativa al menos buena parte de su presentación gráfica en sociedad. Para los clubes, apelar a su lugar y a su actividad forma parte de una necesidad identificativa. Club Natación Almería, o Club Natació Terrassa, no deja de ser una especie de tarjeta de visita: se trata de entidades (club), inicialmente especializadas en natación desde su origen, ubicada una en Almería y otra en Tarrasa.

El comercio, en cambio, se desenvuelve entre coordenadas más amplias y variadas. Cada establecimiento busca estrategias apropiadas al público al que se dirige, muy vinculadas a su dimensión como empresa. Es el campo preferido de sajonización idiomática, con su conocida y sempiterna apelación a la internacionalización y a la calidad de sus productos. Los minoristas más especializados, por su parte, acuden a apelaciones más 
directas, como el expresivo Basket de Terrassa.

En tercer y último lugar, las zonas y barriadas urbanas influyen en las rotulaciones deportivas, aunque menos de lo que cabría pensar en principio. Es cierto que el inglés tiende a abundar más en las zonas de primor comercial, sobre todo en los centros urbanos. Pero también es verdad que la influencia de la localización ha actuado de manera subordinada a otros factores. Cuando no ha habido condicionamientos de tema, o de necesidad de afirmar una identidad local, entonces sí que la zona ha sido activa y determinante. No en vano el lenguaje del cartel que cuelga en la fachada está antes que el escaparate.

Los resultados de la investigación empírica tampoco han dejado de apuntar hacia algunas de las cuestiones teóricas candentes en relación al PL. Su delimitación nocional, en sentido amplio, está dentro de la sociolingüística, como había consignado la bibliografía hasta ahora. No deja de ser un modo de interacción del lenguaje con la sociedad, por lo que cabe situar al $P L$ dentro de ese cada vez más amplio modelo de la lingüística contemporánea. Cosa distinta será ubicarlo en unas coordenadas con una delimitación exacta, y a la vez, exhaustiva. Ha sido relativamente habitual vincularlo con la planificación lingüística, incluso haciéndolo depender de ella. Pero lo cierto es que esos nexos solo ocurren en parte de la potencial casuística de estas investigaciones. Solo cuando hay una política lingüística explícita en favor de procesos de normalización de una lengua minoritaria se legislan y aplican normativas en esa dirección, dentro de medidas más amplias para favorecer su expansión. Como se ha comprobado aquí en el caso de Tarrasa, su repercusión sobre el $P L$ no siempre es directa ni está exenta de verse condicionada por otros factores y tendencias estandarizadoras, nacionales e internacionales. La legislación lingüística no siempre basta para construir por sí sola un $P L$, al menos a tenor de los datos que aquí se han examinado. De todas formas, la preocupación por la rotulación pública no deja de ser un apartado colateral de la planificación lingüística que, en cambio, ha centrado el grueso de sus esfuerzos en la normativización de la gramática, el vocabulario y la ortografía, la escolarización, o el acceso a 
los medios de comunicación y los estilos formales. Fuera de ese supuesto, en otras comunidades multilingües, o en contextos monolingües, el $P L$ también existe en lugares como Almería o Łódź. Por lo tanto, parece prudente conferir a la planificación un papel más restringido como agente de parte del $P L$ de algunas comunidades concretas, pero no como el gran marco de referencia entre el que se inserta.

Más ocasionalmente se han establecido vínculos con algunos planteamientos de Labov y Milroy. Sin embargo, no parecen más sólidos que los anteriores. Toda la problemática vinculada al $P L$ cae muy lejos de sus fundamentos teóricos y de su impresionante bagaje empírico. No así de parte de su metodología. El variacionismo ha demostrado fehacientemente que las comunidades lingüísticas no son nunca un todo homogéneo. El PL no es, desde luego, una excepción a esa regla de completo fundamento empírico. Existen factores que condicionan la presencia de las lenguas y los fenómenos lingüísticos que las manifiestan dentro del espacio urbano. Franco Rodríguez ha hecho aportaciones decisivas en esa dirección, que es conveniente continuar. Es probable que surjan nuevos factores, entre los que no resulta descabellado pensar en las redes sociales en las que se inscriben las manifestaciones de $P L$, en línea con las ideas de Milroy.

Todas estas cuestiones, en su conjunto, considero que tienen mejor acomodo dentro de la moderna sociolingüística interaccional, si se prefiere, en el marco de la etnografía del habla clásica; o lo que viene a ser lo mismo, en el estudio de la cotidianidad idiomática de las sociedades. De hecho, los métodos aplicados en sus investigaciones, hasta el momento, están más próximo a la raíz etnolingüística de Hymes, Gumperz y sus seguidores que a la de ninguna otra escuela sociolingüística norteamericana. En todo caso, preferiría abogar por una perspectiva más integral que, desde ese fondo interaccional, no desdeñase la importancia metodológica en primera instancia -descriptiva en última- que puede tener incorporar algunos elementos del variacionismo en la línea que acabo de comentar. Sin olvidar, por lo demás, que su explicación última requerirá acudir al contexto semiótico global, en conexión con otros signos no verbales, más 
en sintonía por tanto con la concepción italiana de la sociolingüística.

$\mathrm{Si}$ se alcanza esa mayor estabilidad teórica que reclamaba Shibata, probablemente sea más factible solventar otra de las grandes cuestiones pendientes del $P L$. Me refiero a la definición de las unidades de análisis. La opción más habitual ha sido optar por situarla en el signo. Evidentemente, el $P L$ está compuesto por signos, solo que este es un concepto muy abarcador en las ciencias sociales, no solo en la propia lingüística. Los signos verbales allí conviven con otros, numerosos y diversos. Naturalmente, fuera de las ciudades el universo está repleto de signos también. Por lo tanto parece más conveniente precisar de qué signo concreto se trata. Ya he señalado que la propuesta de Backhaus (2006: 55) y Franco (2005), acotando las unidades de análisis como textos, me parece una aproximación más ajustada al objeto de estudio que persigue el $P L$. Solo que, a partir de ahí, es conveniente seguir avanzando para empezar, en las funciones que cumplen, o que aspiran a cumplir. También apunta la bibliografía que existen rótulos meramente informativos, junto a otros con responsabilidades simbólicas. En ocasiones se introducen puntualizaciones acerca de su capacidad connotativa y yo me permitiría agregar funciones persuasivas, muy vinculadas sobre todo a los establecimientos que toca este trabajo, los comerciales. Por lo demás, esas funciones tampoco han de ser exclusivas, sino que pueden simultanearse en un mismo texto. Desde luego, los emisores cuentan con las funciones a las que están destinados los nombres de sus establecimientos, circunstancia que termina remitiendo a la gran referencia por antonomasia de la etnografía del habla. Participantes y fines son dos componentes del evento comunicativo, tal y como lo diseñó Hymes (1972) en su formulación canónica. Los nombres de los establecimientos, el resto de la cartelería, las inscripciones públicas, cualquier manifestación de $P L$ son, en efecto, textos. Solo que dentro de eventos comunicativos, entre los que se incluye el texto como uno de sus componentes.

Pero eso, en todo caso, esas cuestiones quedan para otra investigación, para seguir indagando en ese apasionante hecho de que el 
lenguaje contribuya a determinar un paisaje urbano, tan complejo como nuestro tiempo.

\section{REFERENCIAS BIBLIOGRÁFICAS}

ÁGUILA SOTO, C. (2005). El ocio en el marco sociocultural de la posmodernidad. Una aproximación desde las prácticas de los jóvenes universitarios. Almería: Universidad de Almería.

BACKHAUS, P. (2006). "Multilingualism in Tokyo: A Look into the Linguistic Landscape". International Journal of Multilingualism $3.1,52-66$.

(2007). Linguistic landscapes: a comparative study of urban multilingualism in Tokyo. Clevedon: Multilingual Matters.

BARTHES, R. (1964). Elementos de Semiología. Madrid: A. Corazón.

BEN-RAFAEL, E. et alii (2006). "Linguistic Landscape as Symbolic Construction of the Public Space: The Case of Israel". International Journal of Multilingualism 3.1, 7-30.

BLÁZQUEZ, S. (2015). "El deporte inyecta salud a la economía". El Pais, 21/12 (http://economia.elpais.com/economia/2015/02/20/ actualidad [15/11/2016]).

CASTILLO LLUCH, M. y SÁEZ RIVERA, D. M. (2011). “Introducción al paisaje lingüístico de Madrid”. Lengua y migración 3.1, 73-88.

GOBIERNO DE ESPAÑA (2012). Clasificación Internacional de Productos y Servicios Niza. Madrid: Ministerio de Industria, Energía y Turismo.

ECO, U. (1964). Apocalípticos e integrados. Lumen: Barcelona, 1965. (1968). La estructura ausente. Lumen: Barcelona, 1974.

FRANCO RODRÍGUEZ, J. M. (2005). "El español en el condado de Los Ángeles desde la señalética comercial y urbana”. Hispania 88.4, 825-833.

(2011). "Linguistic Landscape and Language Maintenance: 
The Case of Los Angeles and Dade Counties". En Culture and Language: Multidisciplinary Studies, Michael A. Morris (ed.), 69119. Frankfurt: Lang.

(2013). "An Alternative Reading of the Linguistic Landscape:

The Case of Almería". Revista Internacional de Lingüística Iberoamericana XI.21, 109-134.

GAMONAL, R. (2011). “GRAPHÓPOLIS: Gráfica y tipografía urbana en Madrid". Actas Icono 14. 8, 1-11.

GARCÍA FERRANDO, M. (1986). Hábitos deportivos de los españoles. Sociología del comportamiento deportivo. Madrid: Consejo Superior de Deportes.

GONZÁLEZ RIAZA, B. (2006). "La ciudad escrita”. Paperback 2.

GREENDORFER, S. H. (1981). Sociology of sport: diverse perspective. West Point: Leisure Press.

GREIMAS, A. J. (1976). Sémiotique et sciences sociales. Paris: Seuil.

HUEBNER, T. (2006). "Bangkok's linguistic landscapes: Environmental print, code mixing and language change". International Journal of Multilingualism 3.1, 31-51.

LANDRY, R. \& BOURHIS, R. Y. (1997). "Linguistic Landscape and Ethnolinguistic Vitality An Empirical Study”. Journal of Language and Social Psychology 16.1, 23-49.

LOTMAN, Y.(1973-1976). Semiótica de la cultura. Madrid: Cátedra,1979. (1984). La semiosfera, Madrid: Cátedra, 1988.

MUÑOZ CARROBLES, D. (2010). "Breve itinerario por el paisaje lingüístico de Madrid”. Ángulo Recto 2.2, 103-109.

PONS RODRÍGUEZ, L. (2012). El paisaje lingüístico de Sevilla. Lenguas $y$ variedades en el escenario urbano hispalense. Sevilla: Diputación Provincial de Sevilla.

SEBBA, M. (2010). "Book review: 'Linguistic Landscapes: A Comparative Study of Urban Multilingualism in Tokyo'”. Writing Systems Research 2.1, 73-76.

SERRANO, S. (1979). Signes, llengua i cultura. Barcelona: Edicions 62. 
(1993). Comunicació, llenguatge i societat. Barcelona: Empúries.

SHOHAMY, E. \& GORTER, D. (2009). Linguistic landscape: Expanding the scenery. Nueva York / Londres: Routledge.

SWEETSER, F. L. (1965). "Factor Structure as Ecological Structure in Helsinki and Boston". Acta Sociologica 8, 205-225.

TIMMS, D. W. G. (1971). The Urban Mosaic. Cambridge: C.U.P.

Recibido el 11 de enero de 2018.

Aceptado el 3 de abril de 2018. 